Available online on 15.10 .2020 at http://jddtonline.info
Open Access to Pharmaceutical and Medical Research
unrestricted non-commercial use, provided the original work is properly cited

Open Access

Research Article

\title{
A Prospective Observational Study on Risk Factors and Prescribing Patterns of Drugs used in Stroke Patients at a Tertiary Care Teaching Hospital.
}

\author{
Hussainy Syed Areefulla*, Habeeb Madiha, Jeelani Ayesha, Sultana Sumaiya, Meeran Mohd Mohiuddin \\ Department of Pharmacy Practice, Mesco College of Pharmacy, Hyderabad, Telangana, India
}

\begin{abstract}
Objectives: The main aim of the study is to identify the different risk factors, prescribing patterns of medication and rationality of the prescription by observing the management of stroke patients carefully.

Methodology: A prospective and observational study was carried out for a period of 6 months in an in-patient department of a tertiary care hospital. Patients above 18 years of age, patients of either sex and patients who previously had stroke were included. Children below 18 years, pregnant women, AIDS patients, patients unable to respond to verbal questions with no caretakers and patients with head injury were excluded.

Results: A total of 100 prescriptions were analyzed during the 6 months study period. This was found to be higher in males (79) and females (21). The higher risk with notable occurrence in the age group of 60-79 years (43\%). Out of these study populations, (68\%) experienced Ischemic stroke and (32\%) experienced Hemorrhagic stroke. The major risk factors were Hypertension (62\%), alcoholics (41\%). Smoking (33\%) and Diabetes (24\%). Among the 100 prescriptions, Atorvastatin was highly prescribed (96) followed by Pantoprazole (92), Manni tol (64). Out of 100 prescriptions, (67) were rational and (33\%) were irrational.

Conclusion: The prescribing patterns of drug should be based on specificity and severity of stroke in order to facilitate rational use of drugs providing optimal care. Early management of hypertension and diabetes may reduce risk of stroke. Cessation of smoking and alc ohol place a key role in prevention of stroke.
\end{abstract}

Keywords: Ischemic stroke, hemorrhagic stroke, hypertension, diabetes.

Article Info: Received 07 Aug 2020; $\quad$ Review Completed 17 Sep 2020; $\quad$ Accepted 29 Sep 2020; $\quad$ Available online 15 Oct 2020

Cite this article as:

Hussainy SA, Habeeb M, Jeelani A, Sultana S, Meeran MM. A Prospective Observational Study on Risk Factors and Prescribing Patterns of Drugs used in Stroke Patients at a Tertiary Care Teaching Hospital, Journal of Drug Delivery and Therapeutics. 2020; 10(5-s):71-75 http://dx.doi.org/10.22270/jddt.v10i5-s.4469

*Address for Correspondence:

Hussainy Syed Areefulla, 13-5-741, Mustaidpura, Karwan Road, Hyderabad- 500006, India

\section{INTRODUCTION}

Stroke is defined by the World Health Organization (WHO) as a clinical syndrome consisting of rapidly developing clinical signs of focal (or global in case of coma) disturbance of cerebral function lasting more than 24 hours or leading to death with no apparent cause other than a vascular origin. ${ }^{1}$

Stroke is a major cause of death and disability in India. The estimated adjusted prevalence rate of stroke range, 84$262 / 100,000$ in rural and 334-424/100,000 in urban areas. The incidence rate is $119-145 / 100,000$ based on the recent population-based studies. There is also a wide variation in case fatality rates with the highest being $42 \%$ in Kolkata. 2 . The burden of stroke is heterogeneous and is greater among the elderly, men, and African-Americans. 3. In China, 1.3 million people have a stroke each year and $75 \%$ live with varying degrees of disability as a result of stroke. 3
Age, gender, race, ethnicity, and heredity have been identified as markers of risk for stroke. Although these factors cannot be modified. Some of the common modifiable risk factors are High blood pressure, Tobacco use, Diabetes, High cholesterol, Atrial fibrillation or other heart diseases, Certain blood disorders, Transient ischemic attacks, Cigarette smoking, Alcohol, Illicit drug abuse, Various lifestyle factors have been associated with increased stroke risk. Obesity has been associated with higher levels of blood pressure, blood glucose, and atherogenic serum lipids, which are independent risk factors for stroke. ${ }^{3}$

Ischemic stroke (caused by blood clots), Transient ischemic attack (TIA) (a "mini-stroke," caused by a temporary blood clot), Hemorrhagic stroke (caused by ruptured blood vessels) are the three main types of stroke. ${ }^{3}$

Ischemic stroke is an acute episode of focal cerebral, spinal, retinal dysfunction caused by infarction of central nervous 
system tissue. Specifically, ischemic stroke includes embolic infarct, Thrombotic infarct and lacunar infarct. Transient Ischemic Attack (TIA) is a transient event, where arterial blockage in brain resolves on its own without causing any tissue death. Hemorrhagic stroke is an acute episode of focal or global cerebral or spinal dysfunction caused by intraparenchymal, intraventricular, or subarachnoid hemorrhage. Hemorrhagic stroke is of two types mainly; Intracerebral hemorrhage (ICH), Subarachnoid Hemorrhage (SAH). ${ }^{3}$

The study is carried out to create awareness among the physicians, nursing staff and patients regarding its risk factors and management. To assess the overall understanding of the drugs prescribed such as (Antiplatelets, Hypolipidemic drugs, diuretics, anticoagulants) and effectiveness of current inpatient stroke education practice by using the data from the patients and their caretakers. To focus on stroke patients and their caretakers by providing proper information on risk factors of the disease, early management and lifestyle modifications especially during post-stroke care at a tertiary care teaching hospital.

\section{MATERIALS AND METHODS}

A prospective and observational study was conducted at the department of General medicine, Osmania General Hospital. All the patients attending the department of general medicine with the complaints of stroke. The study was conducted for a period of 6 months on 100 patients diagnosed with stroke.

\section{Inclusion criteria}

Patients above 18 years of age were included in the study, patients of either sex, patients with history of stroke.

\section{Exclusion criteria}

Children below 18 years, pregnant women, HIV or AIDS Patient, patients who refused to be the part of the study, patients unable to respond to verbal questions with no caretakers, patient with head injury.

All the relevant and necessary data have been collected from patient's case notes, patient's prescription prescribed by the physician. Laboratory reports, Interviewing patient or patient caretaker (s) and health care professionals.

All the patients admitted at Osmania General Hospital were screened for the stroke. Each subject's detailed history regarding age, sex, weight, socioeconomic status, rural or urban, history of hypertension, history of stroke and its treatment given, social habits and any comorbid conditions to be collected.

The selected patients were observed for signs and symptoms, presence of hemorrhage if any in the cerebrovascular region by CT Scan, motor functioning and improvement in the symptoms following the treatment.

\section{RESULTS}

In this study a total no. of 100 patients were studied during a period of six months. Out of 100 patients it was found that the majority of number of patients was male patients with $79 \%$ whereas the female patients constituted $21 \%$ of the total population as shown in figure 1 .

\section{GENDER DISTRIBUTION OF PATIENTS}

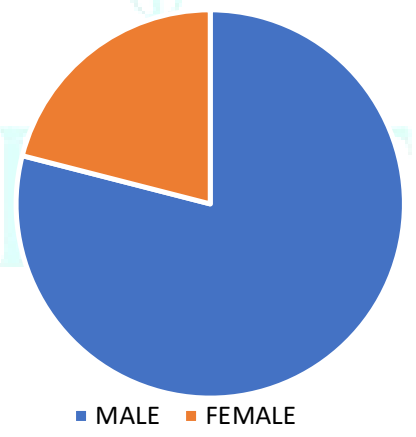

Figure 1: Gender distribution of the patient.

In the age distribution of the patients, it was found that the incidence of stroke was maximum in the age group 60-79 years which comprised of (43\%) patients followed by the age group of 40-59 years which comprised of (40\%) of the entire study population as shown in table 1.

Table 1: Age distribution of the patients.

\begin{tabular}{|l|l|l|l|}
\hline Age of the Patients & Male & Female & Total (\%) \\
\hline $20-39$ & 10 & 3 & $13(13 \%)$ \\
\hline $40-59$ & 31 & 9 & $40(40 \%)$ \\
\hline $60-79$ & 37 & 6 & $43(43 \%)$ \\
\hline$>80$ & 1 & 3 & $4(4 \%)$ \\
\hline
\end{tabular}


Out of the study population, $68 \%$ patients were diagnosed as ischemic stroke and $32 \%$ were diagnosed as hemorrhagic stroke as shown in figure 2 .

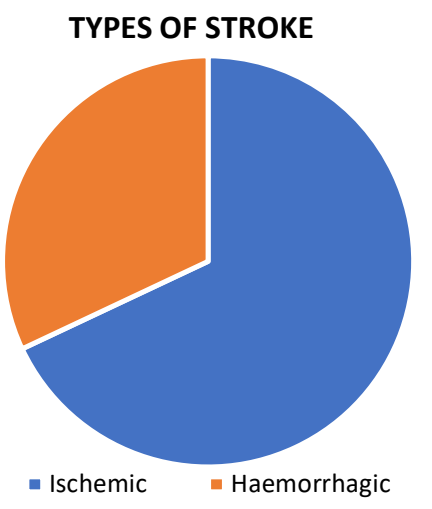

Figure 2: Types of stroke.

Out of the total study population, the majority of the subjects had a risk factor of hypertension, followed by Alcohol, smoking, diabetes and other factors as shown in table 2.

Table 2: various risk factors and co-morbidities associated with the stroke patients.

\begin{tabular}{|l|l|}
\hline RISK FACTORS & NUMBER OF PATIENTS (\%) \\
\hline HYPERTENSION & $62(62 \%)$ \\
\hline ALCOHOL & $41(41 \%)$ \\
\hline SMOKING & $33(33 \%)$ \\
\hline DIABETES & $24(24 \%)$ \\
\hline EPILEPSY & $5(5 \%)$ \\
\hline ASSOCIATED CARDIAC PROBLEMS & $7(7 \%)$ \\
\hline PULMONARY DISEASES & $3(3 \%)$ \\
\hline OTHERS & $14(14 \%)$ \\
\hline OLD CVA & $8(8 \%)$ \\
\hline
\end{tabular}

Most common drug prescribed in hospitalized stroke patients were Supplements, Antibiotics, Antacids, followed by Antihypertensive agents and Lipid lowering agents as shown in the table 3 and figure 3 representing the graph.

Table 3: Groups of drugs prescribed in stroke patients.

\begin{tabular}{|l|l|}
\hline GROUP OF DRUGS & $\begin{array}{l}\text { Number of drugs prescribed } \\
\text { to patients with (\%) }\end{array}$ \\
\hline Supplements & $111(13.48 \%)$ \\
\hline Antibiotics & $107(13.00 \%)$ \\
\hline Antacids & $106(12.87 \%)$ \\
\hline Antihypertensives & $101(12.27 \%)$ \\
\hline Lipid Lowering agents & $96(11.66 \%)$ \\
\hline Others & $77(9.35 \%)$ \\
\hline Antiplatelet & $73(8.86 \%)$ \\
\hline Diuretic & $70(8.50 \%)$ \\
\hline Antiemetic & $27(3.28 \%)$ \\
\hline Antiepileptic & $25(3.03 \%)$ \\
\hline Antidiabetic & $22(2.67 \%)$ \\
\hline Anticoagulant & $8(0.97 \%)$ \\
\hline
\end{tabular}




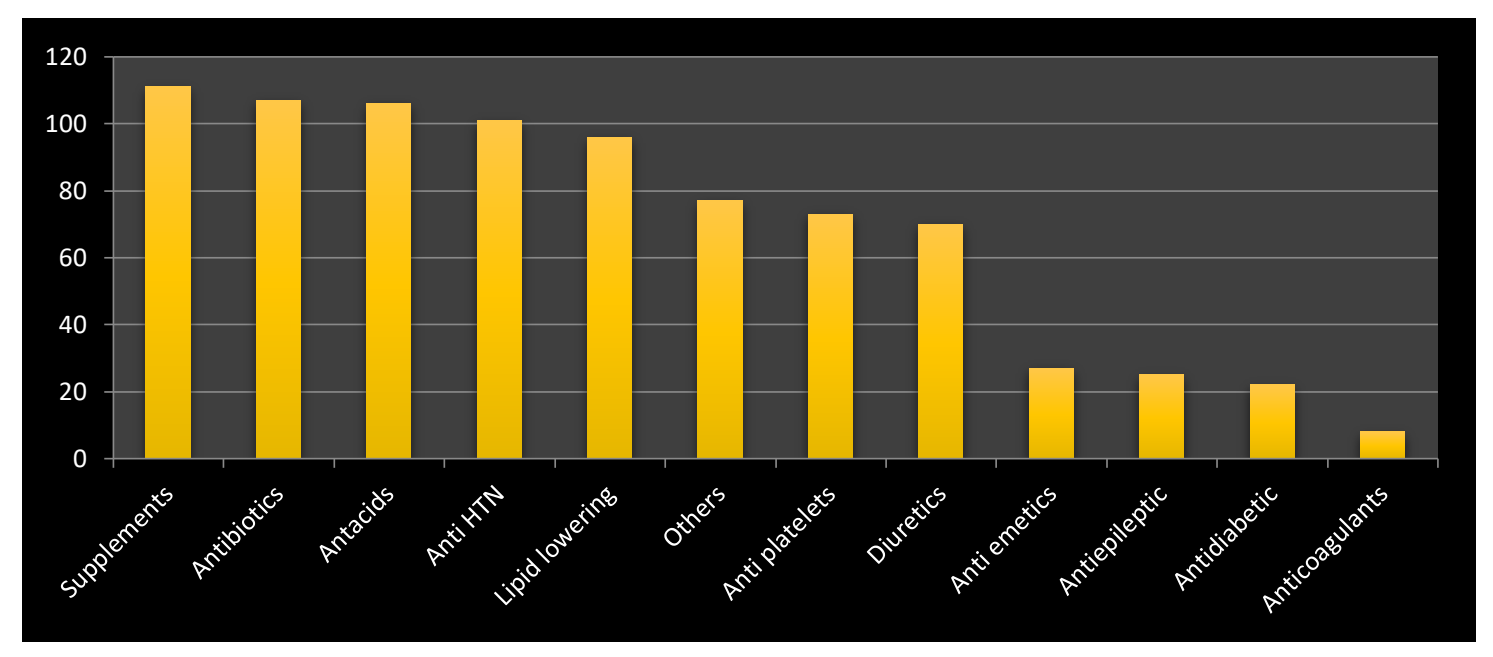

Figure 3: Groups of drugs prescribed to the patients.

The most common drugs prescribed in hospitalized stroke patients were Supplements, Antibiotics, Antacids followed by Antihypertensive agents and Lipid lowering agents as shown in table 4.

Table 4: Individual agents in each category of drugs prescribed in patients.

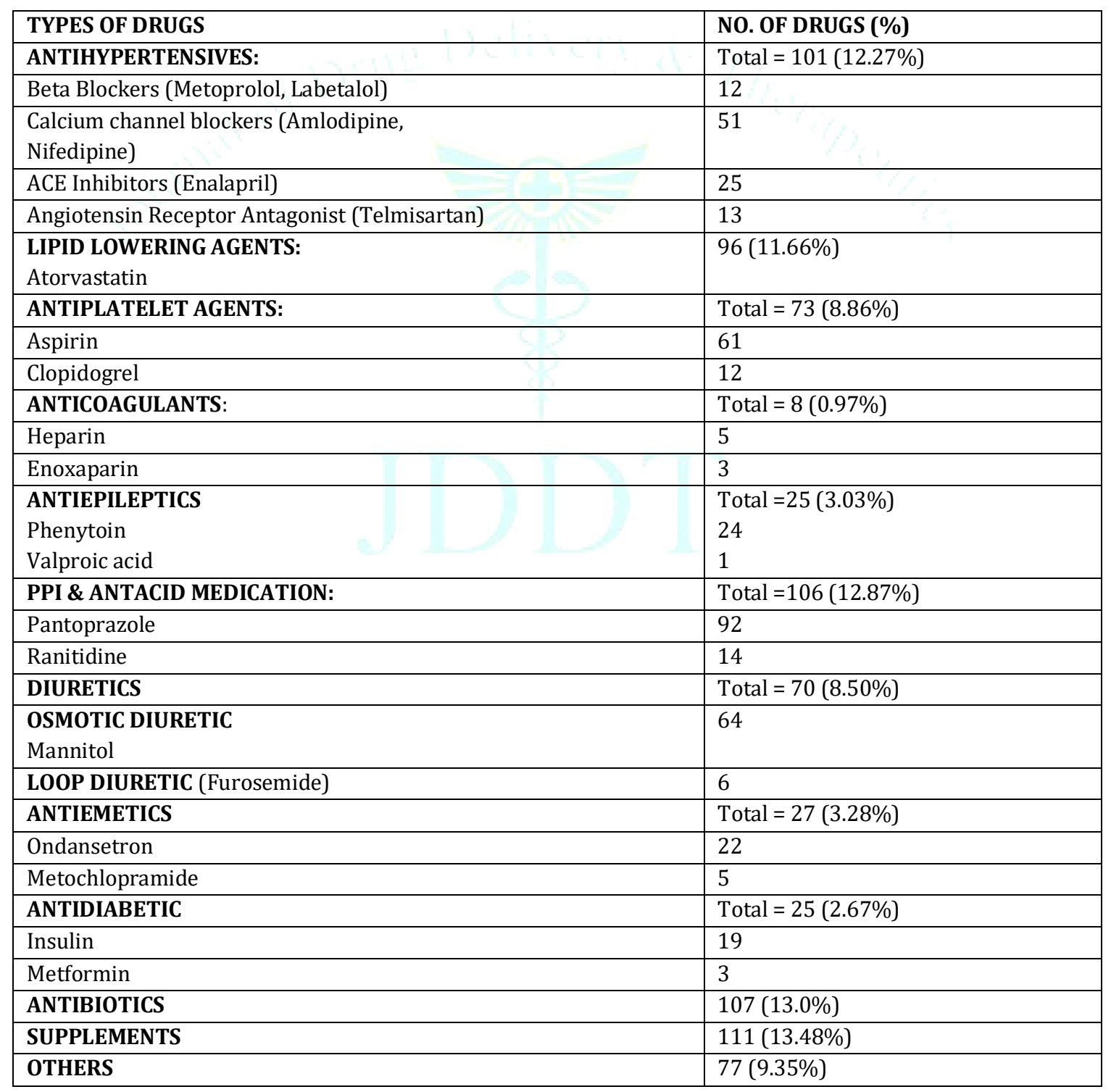

Out of total prescriptions the majority of prescriptions were rational which is $67 \%$ and remaining were irrational which is $33 \%$ as shown in table 5 . 
Table 5: rationality of the prescription.

\begin{tabular}{|l|l|l|}
\hline Rationality & No. of Prescriptions & Percentage \\
\hline Rational & 67 & $67 \%$ \\
\hline Irrational & 33 & $33 \%$ \\
\hline
\end{tabular}

\section{DISCUSSION}

An observational study was carried out on risk factors and prescribing patterns of drugs used in stroke patients. Out of 100 stroke patients, $79 \%$ were males and $21 \%$ were females which is similar to the previous study conducted by nikhat et.al (2018). 4

The incidence of stroke was high among the age group of 6079 years $(43 \%)$ followed by $40-59$ years of age $(40 \%)$ which is similar to the previous study conducted by K. Soumya Lakhsmi et.al (2018), stating that stroke is more common among the age group of $40-80$ years. ${ }^{5}$

After examining the subjects based on the types of Strokes, it was found that majority of the patients suffered an ischemic stroke $(68 \%)$ followed by hemorrhagic stroke (32\%) which is similar to a previous study conducted by Mohammed Yaseen Abbasi et.al $(2012)^{6}$ stating that most of the patients suffered an ischemic stroke.

In the present study, it was found that most of the subjects were hypertensives (62\%) which is a major risk factor for development and progression of stroke followed by alcoholics (41\%), addicted to smoking (33\%) \& diabetics (24\%) which is in accordance with other studies conducted by Spurthi et.al (2016) 7 , stating that most of the subjects with stroke are hypertensive.

After analyzing the overall list of medications that were prescribed among 100 prescriptions, it was found that the most consumed category of drugs were Supplements (13.48\%), Antibiotics (13.0\%), Antacids / PPI ( $12.87 \%)$, followed by Antihypertensive agents (12.27\%) and Lipid lowering agents (11.66\%). For Risk Factors like Hypertension, Calcium channel blockers like amlodipine were the most prescribed followed by ACEIs like enalapril and ARBs like Telmisartan followed by Beta Blockers like Metoprolol, Labetolol, Propanolol. The prescribing patterns of drugs revealed that among the number of 100 prescriptions, Atorvastatin was highly prescribed drug (96) followed by Pantoprazole (92), Mannitol (64), Aspirin (61) and Calcium channel blockers: Amlodipine/Nifedipine (51), and others in accordance with other studies conducted by subhransu et.al (2018). 8

The present study reveals the prescribing pattern of drugs used in the treatment of stroke stating that most of the subjects were admitted with ischemic stroke followed by Hemorrhagic stroke in accordance with previous study conducted by vurumadla et.al (2014). ${ }^{9}$. Drugs prescribed for the treatment of stroke were according to AHA/ASA guidelines. Antihypertensive were the major class of drugs preferred for the improvement of risk factors as they lower the blood pressure in hemorrhagic stroke and lowers the risk of recurrent stroke. Lipid Lowering Agents and Antiplatelet drugs were the most common drugs used for prevention of clot formation.

Out of 100 prescriptions, $67 \%$ were rational as they were given in accordance with the AHA/ASA guidelines and 33\% were irrational due to the number of severe drug interactions between them.
According to the AHA/ASA guidelines, CT/MRI are the standard diagnostic test for the detection of different type of stroke performed at the time of admission in the ER at the study site.

\section{CONCLUSION}

In the present study, a sample size of 100 stroke patients were evaluated based on the prescribing pattern of drugs used in the treatment of different types of stroke stating that most of the subjects were admitted with Ischemic stroke. In our study, we observed that the incidence of stroke was more common in males than females. Severity of stroke was based on, risk factors and symptoms. Various classes of drugs prescribed were Antihypertensives, Lipid lowering Agents, Antiplatelets, Diuretics, Antiemetics, Antiepileptics, Antidiabetics, Anticoagulants, Supplements, Antibiotics, Antacids. Drugs prescribed for the treatment of stroke were according to AHA/ASA guidelines. Antihypertensives, Osmotic Diuretic, Lipid Lowering agents, Antiplatelets were the major class of drugs preferred for the improvement of symptoms. Atorvastatin was the most commonly prescribed drug for treating dyslipidemia.

CT/MRI which is a standard test for the diagnosis of Stroke was performed in this study, according to AHA/ASA guidelines. In our study, we found that there were few severe drug interactions by co-administering the drugs like (Aspirin + Enalapril) it is advisable to change the time of Administration. The drugs (Nifedipine + Phenytoin) resulted in severe drug interactions therefore it is advisable to withdraw the medication or use of alternate drug. It was found that $33 \%$ of prescriptions were irrational which has shown atleast one serious drug-drug interaction potentially leading to renal impairment. Majority of the prescriptions were rational (67\%) and were in accordance with the AHA/ASA guidelines.

The findings in our study stress the need for early and appropriate management of stroke to prevent further complications of stroke. Physiotherapy, lifestyle changes and better management of risk factors have a major effect on recovery of stroke with improve quality of life and symptoms.

\section{REFERENCES}

1. WHO MONICA Project Investigators. The World Health Organization MONICA Project (monitoring trends and determinants in cardiovascular disease). J Clin Epidemiol. 1988; 41(2):105-14.

2. Banerjee TK, fifty years of stroke researches in india, (2010) (journal of stroke).

3. www.ahajournals.org/doi/full/10.1161/01.str.28.7.1507

4. Syeda Rana Nikhat - Study on risk factors and management of stroke at a tertiary care teaching hospital (International Journal of Pharmacy and Pharmaceutical Sciences) (2018) ISSN- 09751491, Vol. 10 Issue 6.

5. Lakhsmi KS, Study on symptoms, risk factors and prescribing patterns in stroke patients. (Indo American journal of pharmaceutical research) (2018) ISSN no. 2231-6876.

6. Abbasi MY, Prescribing pattern of drugs in stroke patients: a prospective study 2012; 3(4):283-288.

7. Spurthi T, Risk Elements and utilization in stroke patients. 2016; 19(7):A864.

8. Jena SK, Utilisation pattern of different drugs in different types of stroke in a tertiary care hospital, international journal of pharmaceutical sciences review and research; 2018; 18:85-90.

9. Vurumadla S, A study on symptoms, risk factors and prescribing pattern of drugs used in stroke patients (indian journal of pharmacy and pharmaceutical sciences) 2014; 7(1):421-6. 